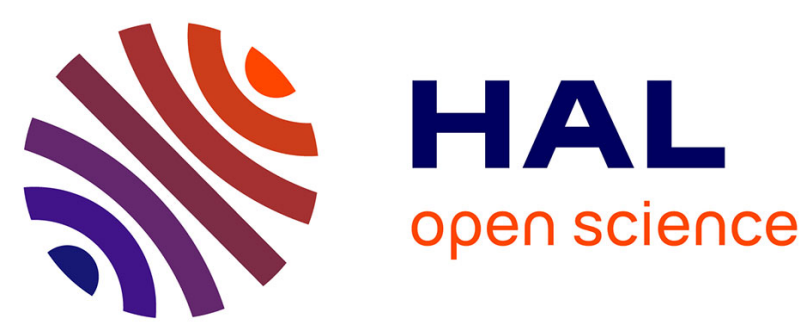

\title{
ÉTUDE QUANTITATIVE PAR EFFET MÖSSBAUER DE LA RADIOLYSE DU SULFATE FERREUX HEPTAHYDRATE FeSO4.7 H2O
}

\author{
C. Kellershohn, F. Soubirou, Cédric Hubert
}

\section{- To cite this version:}

C. Kellershohn, F. Soubirou, Cédric Hubert. ÉTUDE QUANTITATIVE PAR EFFET MÖSSBAUER DE LA RADIOLYSE DU SULFATE FERREUX HEPTAHYDRATE FeSO4.7 H2O. Journal de Physique Colloques, 1974, 35 (C1), pp.C1-73-C1-75. 10.1051/jphyscol:1974123 . jpa-00215498

\section{HAL Id: jpa-00215498 https://hal.science/jpa-00215498}

Submitted on 1 Jan 1974

HAL is a multi-disciplinary open access archive for the deposit and dissemination of scientific research documents, whether they are published or not. The documents may come from teaching and research institutions in France or abroad, or from public or private research centers.
L'archive ouverte pluridisciplinaire HAL, est destinée au dépôt et à la diffusion de documents scientifiques de niveau recherche, publiés ou non, émanant des établissements d'enseignement et de recherche français ou étrangers, des laboratoires publics ou privés. 


\title{
ÉTUDE QUANTITATIVE PAR EFFET MÖSSBAUER DE LA RADIOLYSE DU SULFATE FERREUX HEPTAHYDRATE FeSO $\mathrm{F}_{4} .7 \mathrm{H}_{2} \mathrm{O}$
}

\author{
C. KELLERSHOHN, F. SOUBIROU et C. HUBERT \\ Laboratoire de Biophysique, \\ Faculté de Médecine Necker-Enfants Malades, Paris, France
}

\begin{abstract}
Résumé. - L'irradiation par rayons X d'échantillons polycristallins de $\mathrm{FeSO}_{4} .7 \mathrm{H}_{2} \mathrm{O}$, à l'air libre et à la température ordinaire, entraîne la formation de $\mathrm{Fe}^{3}$ ", probablement sous la forme d'un hydroxyde ferrique d'après le déplacement chimique et le couplage quadrupolaire fourni par la spectrométrie Mössbauer.

Les échantillons de $10 \mathrm{mg}$ de $\mathrm{Fe} / \mathrm{cm}^{2}$ sont irradiés au moyen d'un tube à rayons $\mathrm{X}$ sous tension constante de $50 \mathrm{kV}$ et les spectres réalisés à la température ordinaire au moyen d'un spectromètre à accélération constante et d'une source ${ }^{57} \mathrm{Co}-\mathrm{Pd}$. La variation de la proportion de $\mathrm{Fe}^{3 *}$ en fonction de la dose de radiation est déterminée par comparaison des spectres Mössbauer des échantillons irradiés avec ceux de mélanges étalons de $\mathrm{FeSO}_{4} .7 \mathrm{H}_{2} \mathrm{O}$ et d'un hydroxyde de fer ayant les mêmes paramètres Mössbauer que l'ion $\mathrm{Fe}^{3}$ : résultant de l'jrradiation.

La quantité d'énergie nécessaire à la production d'un ion $\mathrm{Fc}^{3+}$ à partir d'une molécule de $\mathrm{FeSO}_{4} .7 \mathrm{H}_{2} \mathrm{O}$ est déduite des données expérimentales et comparée à celle nécessaire pour obtenir le même effet sur le sulfate ferreux en solution aqueuse. Quelques incidences des résultats obtenus sont discutées.
\end{abstract}

\begin{abstract}
The irradiation by X-rays of polycrystalline samples of $\mathrm{FeSO}_{4} .7 \mathrm{H}_{2} \mathrm{O}$ in a normal atmosphere and at room temperature leads to the formation of $\mathrm{Fe}^{3 \div}$, probably in the form of ferric hydroxide as deduced from the isomeric shift and the quadrupole splitting.

The samples of $10 \mathrm{mg}$ of $\mathrm{Fe} / \mathrm{cm}^{2}$ are irradiated by means of an X-ray tube under constant voltage of $50 \mathrm{kV}$ and the spectra taken at room temperature by a constant acceleration spectrometer equiped with a ${ }^{57} \mathrm{Co}-\mathrm{Pd}$ source. The variation of the proportion of $\mathrm{Fe}^{3}$ according to the dose of radiation is determined by comparison of the Mössbauer spectra of the irradiated samples with those of reference mixtures of $\mathrm{FeSO}_{4} .7 \mathrm{H}_{2} \mathrm{O}$ and of an iron hydroxide having the same Mössbauer parameters as the $\mathrm{Fe}^{3+}$ ion resulting from the irradiation.

The quantity of energy necessary for the production of an $\mathrm{Fe}^{3+}$ ion from a molecule of $\mathrm{FeSO}_{4} .7 \mathrm{H}_{2} \mathrm{O}$ is deduced from the experimental data and compared with the energy necessary to obtain the same effect on ferrous sulfate in aqueous solution. Some implications of the results obtained are discussed.
\end{abstract}

La production de fer ferrique au cours de lirradiation de solutions aqueuses de sulfate ferreux par les radiations ionisantes a fait l'objet d'un nombre considérable de travaux [1] et est à la base de la méthode de dosimétrie chimique de loin la plus utilisée, depuis son introduction par Fricke et Morse [2] il y a plus de quarante ans. 11 semble intéressant par comparaison d'étudier le comportement du sulfate ferreux à l'état solide sous l'action des rayonnements ionisants. Nous avons effectué une telle étude en utilisant la spectrométrie Mössbauer qui, permettant une séparation quantitative et non destructive des fers ferreux et ferrique, nous a paru une méthode de choix.

1. Matériel et méthode. - Nous avons utilisé du sulfate ferreux heptahydrate $\mathrm{FeSO}_{4} .7 \mathrm{H}_{2} \mathrm{O}$ Merck pour analyse. Les échantillons circulaires de poudre cristalline de $1,7 \mathrm{~cm}$ de diamère ont une épaisseur de $10 \mathrm{mg}$ de Fe par $\mathrm{cm}^{2}$.
Ils sont irradiés au moyen d'un tube à rayons $X$ à tension constante et à anticathode de tungstène Baltographe CE $50 / 15$ fonctionnant sous $50 \mathrm{kV}$ et $15 \mathrm{~mA}$. Après contrôle au moyen d'un spectromètre $\mathrm{Si}(\mathrm{Li})$, nous avons adopté comme répartition spectrale du tube celle proposée par Greening [3] pour la tension? et le type d'anticathode utilisés. Connaissant la répartition spectrale, la mesure du débit dexposition au moyen d'une chambre d'ionisation Victoreen pour basse énergie modèle $n^{0} 651$ a permis de déterminer pour ce tube une intensité de $79,4 \times 10^{8} \mathrm{erg} \cdot \mathrm{sr}^{-1} \cdot \mathrm{h}^{-1}$. A cette intensité correspond pour l'échantillon, situé à $r=4,6 \mathrm{~cm}$ du foyer du tube, un débit de dose absorbée de $\mathrm{d} D / \mathrm{d} t=18,75 \mathrm{Mrd} / \mathrm{h}$. Huit nivealux de doses ont été utilisés correspondant à des temps d’irradiation s'échelonnant de 24 à $9 \times 24 \mathrm{~h}$.

Les spectres Mössbauer sont enregistrés à la température du laboratoire au moyen deun dispositif à accélération constante de Thenard et Victor [4] associé at 
un compteur proportionnel Twenty Century PX 13099 et un analyseur multicanaux Intertechnique Didac 800 . L'échantillon muni d'un diaphragme de plomb de $1 \mathrm{~cm}$ de diamètre est situé à là même distance $6 \mathrm{~cm}$ du détecteur et d'une source ${ }^{57} \mathrm{Co}-\mathrm{Pd}$ de $5 \mathrm{mCi}$. La durée d'enregistrement de chaque spectre est de $24 \mathrm{~h}$. Les spectres expérimentaux sont décomposés par la méthode des moindres carrés en courbes lorentziennes au moyen d'un programme d'ordinateur convenable fournissant la largeur et la surface de ces courbes.

2. Résultats. - La figure 1 montre les spectres Mössbauer des échantillons obtenus pour le $\mathrm{FeSO}_{4} .7 \mathrm{H}_{2} \mathrm{O}$ non irradié et les huit niveaux d'irradiation choisis. Le spectre de $\mathrm{FeSO}_{4} .7 \quad \mathrm{H}_{2} \mathrm{O}$ est caractérisé par un déplacement isomérique par rapport au fer $\delta \mathrm{Fe}=1,25 \pm 0,01 \mathrm{~mm}$ et un dédoublement quadrupolaire $\Delta=3,24 \pm 0,02 \mathrm{~mm}$ qui correspondent bien aux valeurs de la littérature. On voit que

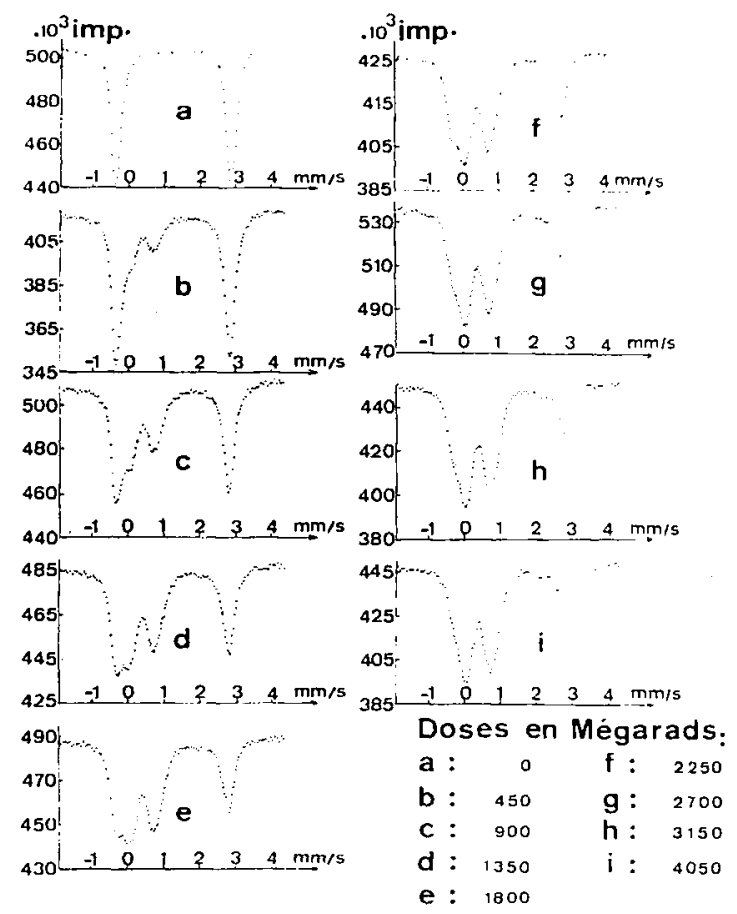

Spectres Mössbauer $\mathrm{FeSO}_{4} \cdot 7 \mathrm{H}_{2} \mathrm{O}$ irradiè par $\mathrm{RX}$

FIG. 1.

pour des doses absorbées croissantes le doublet caractéristique de $\mathrm{FeSO}_{4} .7 \mathrm{H}_{2} \mathrm{O}$ disparaît progressivement au bénéfice d'un doublet de paramètres $\delta \mathrm{Fe}=0,41 \pm 0,01 \mathrm{~mm}$ et $\Delta=0,70 \pm 0,01 \mathrm{~mm}$ caractéristiques de l'apparition d'une structure ferrique. Ceci confirme le fait déjà connu à partir de l'analyse chimique conventionnelle que la décomposition radiolytique de $\mathrm{FeSO}_{4} .7 \mathrm{H}_{2} \mathrm{O}$ s'accompagne de la production de $\mathrm{Fe}^{3+}$ [5]. Ce composé ferrique semble insensible à l'action du rayonnement dans le domaine des doses considérées qui s'étend de 0 à $4000 \mathrm{Mrd}$.

La figure 2 montre la variation du rapport $A_{2} / A_{1}$ des aires des spectres Mössbauer de $\mathrm{Fe}^{3+}$ et $\mathrm{Fe}^{2+}$

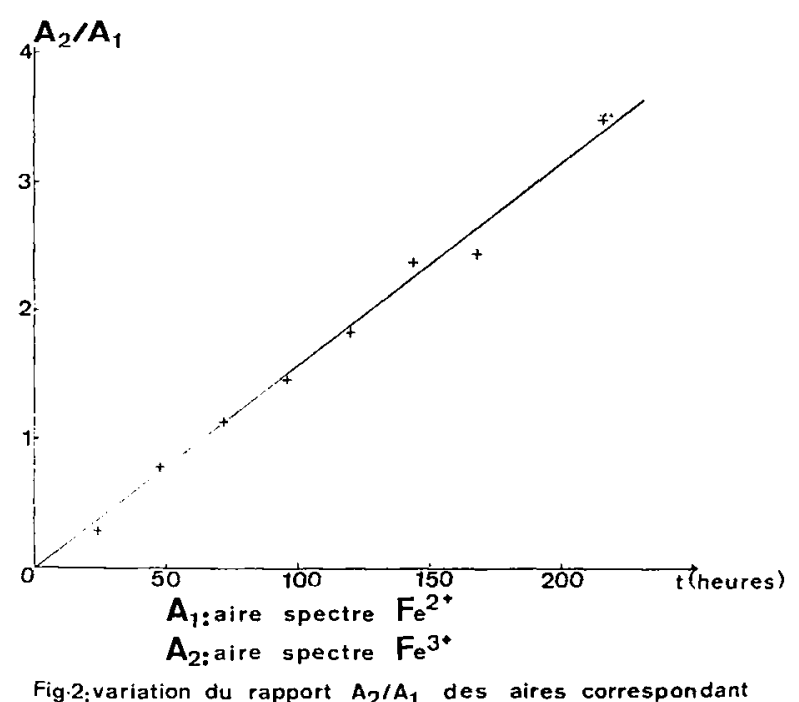

Fig.2:variation du rapport $\mathrm{A}_{2} / \mathrm{A}_{1}$ des aires correspondant
a $\mathrm{Fe}^{3}$ et $\mathrm{Fe}^{2}$ dans le spectre Mössbauer $\mathrm{FeSO}_{4} \cdot 7 \mathrm{H}_{2} \mathrm{O}$ irradié. en fonction du temps $t$ dirradiation.

FIG. 2.

en fonction du temps d'irradiation. Cette variation est linéaire. D'autre part, il est admis [6] que pour des absorbeurs dont l'épaisseur est de l'ordre de $10 \mathrm{mg}$ $\mathrm{Fe} / \mathrm{cm}^{2}$ le rapport des aires est proportionnel au rapport des nombres d'ions $\mathrm{Fe}^{2+}$ et $\mathrm{Fe}^{3+}$ par unité de masse de l'échantillon : $A_{2} / A_{1}=\beta \cdot\left[\mathrm{Fe}^{3+}\right] /\left[\mathrm{Fe}^{2+}\right]$. Nous avons vérifié cette linéarité et déterminé le coefficient $\beta$ en effectuant le spectre Mössbauer d'absorbeurs de $10 \mathrm{mg} \mathrm{Fe} / \mathrm{cm}^{2}$, constitués par des mélanges en proportions variées de $\mathrm{FeSO}_{4} .7 \mathrm{H}_{2} \mathrm{O}$ et d'oxyde de fer sucré Merck. En effet, nous avons pu montrer que ce dernier composé qui se caractérise par des valeurs $\delta \mathrm{Fe}=0,34 \pm 0,01$ et $A=0,71 \pm 0,01$ permettant de le considérer comme un oxyde $\beta$-FeOOH [7], présentait une largeur de raie et un facteur de LambMössbauer égaux aux erreurs expérimentales près à ceux du composé ferrique résultant de l'irradiation de $\mathrm{FeSO}_{4} .7 \quad \mathrm{H}_{2} \mathrm{O}$. La valeur trouvée pour $\beta$ est 1,48 .

En posant $A_{2} / A_{1}=\alpha \beta t$ nous avons

$$
\left[\mathrm{Fe}^{3+}\right] /\left[\mathrm{Fe}^{2+}\right]=\alpha t
$$

et par suite $\left[\mathrm{Fe}^{2+}\right] /[\mathrm{Fe}]=1 / 1+\alpha t$, en désignant par [Fe] le nombre total d'atomes de fer par unité de masse de l'échantillon. Cette dernière relation est caractéristique d'une cinétique du $2^{\mathrm{e}}$ ordre, telle que :

$\frac{\mathrm{d}\left[\mathrm{Fe}^{2+}\right]}{\mathrm{d} t}=-K \frac{\mathrm{d} D}{\mathrm{~d} t}\left[\mathrm{Fe}^{2+}\right]^{2}$ avec $\alpha=K[\mathrm{Fe}] \frac{\mathrm{d} D}{\mathrm{~d} t}$.

Le rendement initial de la radiolyse en nombre de $\mathrm{Fe}^{3+}$ par $100 \mathrm{eV}$ absorbé est alors donné par :

$$
G_{0}=\left[\frac{\mathrm{d}\left[\mathrm{Fe}^{3+}\right]}{\mathrm{d} D}\right]_{t=0}=K[\mathrm{Fe}]^{2}
$$

avec

$$
K[\mathrm{Fe}]=\frac{\alpha}{\mathrm{d} D / \mathrm{d} t}
$$


et $[\mathrm{Fe}]=6,02 \times 10^{23} / 278$ (masse moléculaire de $\mathrm{FeSO}_{4} .7 \mathrm{H}_{2} \mathrm{O}$ ), le débit de dose étant exprimé en $100 \mathrm{eV} \cdot \mathrm{g}^{-1}$. La détermination de la pente $\alpha \beta$ de la droite expérimentale de la figure 2 fournit

$$
\alpha=\alpha \beta / \beta=0,0156 / 1,48=0,0113 \mathrm{~h}^{-1}
$$

et $G_{0}=1,94$ molécule par $100 \mathrm{eV}$ absorbés.

3. Discussion. - Nous n'avons pas actuellement d'interprétation du fait que la radiolyse de $\mathrm{FeSO}_{4} .7 \mathrm{H}_{2} \mathrm{O}$ paraît suivre une cinétique du $2^{e}$ ordre. Notons néanmoins à ce sujet que d'après Johnson [5] l'ion ferrique, l'ion sulfite et l'hydrogène constituent les produits de radiolyse de $\mathrm{FeSO}_{4} .7 \quad \mathrm{H}_{2} \mathrm{O}$ et que stœchiométriquement deux ions ferriques doivent être trouvés pour chaque $\mathrm{H}_{2}$ et ion sulfite formés.

La valeur de $G_{0}=1,94$ ion $\mathrm{Fe}^{3+}$ produit par $100 \mathrm{eV}$ absorbés est voisine de celle de 1,8 obtenue par Johnson [5] par analyse chimique conventionnelle. Elle est sensiblement différente de celle de 1,15 que l'on peut déduire de données fournies par Wertheim et Buchanan [8]. On voit qu'elle est respectivement huit fois et quatre fois plus petite que celle de 15,6 et 8,2 correspondant aux solutions de sulfate ferreux aérées et privées d'oxygène classiquement utilisées en dosimétrie chimique [1].

Notons enfin que $4000 \mathrm{Mrd}$ correspondent à un épuisement de $80 \%$ environ de $\mathrm{FeSO}_{4} .7 \mathrm{H}_{2} \mathrm{O}$, ce qui permet d'envisager une méthode de mesure des doses très élevées.

\section{Bibliographie}

[1] FrICKe, H. et Hart, E. J., Radiation Dosimetry, Vol. II, Instrumentation, edited by F. H. Attix et W. C. Roesch (Academic Press, New York and London) 1966.

[2] Fricke, H. et Morse, S., Am. J. Roentgenol. Radium Therapy Nucl. Med. 18 (1927) 430.

[3] Greening, J. R., Topics in Radiation Dosimetry', Suppl. 1, edited by F. H. Attix (Academic Press, New York and London) 1972.
[4] Thenard, J. et Victor, G., Nucl. Instr, and Meth. 93 (1971) 311.

[5] Johnson, E. R., J. Phys. \& Chem. Solids 78 (1956) 5196.

[6] Yoshroka, T., Gohshi, Y. et Konno, H., Anal. Chem. 40 (1960) 603.

[7] MeiseL, W. et KreYsa, G., Z. anorg. allg. Chem. 395 (1973) 31.

[8] Wertheim, G. K., Buchanan, D. N. E., Chem. Phys. Lett. 3 (1969) 87. 